\title{
Feed-Forward Technique to Measure the Reflection Coefficient Under CW High-Power Signals
}

\author{
Carlos Collado, Senior Member, IEEE, Marta González-Rodríguez, Student Member, IEEE, José M. González- \\ Arbesú, Jordi Mateu, Senior Member, IEEE, Jordi Verdú, and Alberto Hueltes
}

\begin{abstract}
This paper describes a measurement setup to obtain the reflection coefficient response over the frequency of a given device when this is subject to a single tone high-power signal. The proposed system is based on a feed-forward technique to cancel the reflected high-power signal before reaching the vector network analyzer. That allows measuring the effects of the highpower interfering signal into the device's low-power frequency response. This technique does not require either power-calibrated standards or complex calibration techniques. Experiments in several devices are also reported to validate the suitability of this technique.
\end{abstract}

Index Terms - cancellation system, feed-forward, high-power, S-parameters.

\section{INTRODUCTION}

$\mathrm{P}$ ASSIVE intermodulation requirements, power handling of transmitting filters, thermal stability behavior of duplexers, interference blockers and others, are few examples of the increasing interest in characterizing passive devices when they are subject to high-power signals. Many of those devices, for example, a radar protection circuit of jamming signals incorporating frequency-selective power limiters, must retain the low signal performance while dealing with high-power interfering signals. Those signals may fall inside or outside the operation frequency band [1], and therefore the device need to be designed and characterized under those scenarios.

For example, measuring the reflection coefficient of a simple reflective limiter at the limiting state is not trivial with a traditional vector network analyzer (VNA). External highpower amplifiers, isolators, couplers and attenuators must be used, and calibrations need to be performed at each power level of the interfering signal. In addition, the number of VNA's ports is often higher than the number of ports of the device to be characterized [2] because, for example, a reference of the high-power input signal is taken apart of the reflected signal.

Manuscript received February 2018. This work was supported in part by the Spanish Gov. through grants TEC2017-84817-C2-2-R, TEC2017-88343-C42-R, EUIN2017-88928.

C. Collado, M. González-Rodríguez, J.M. González-Arbesú, J. Mateu are with the Signal Theory and Communications Dept. from Universitat Politècnica de Catalunya (UPC), Barcelona 08034, Spain (e-mail: collado@tsc.upc.edu). J. Verdú is with the Universitat Autònoma de Barcelona.
To evaluate the performance of high power devices, techniques such as hot S-parameters and X-parameters [3] have been widely used. The former is typically used to characterize power amplifiers (stability and distortion), and the latter allows for a complete nonlinear characterization of a two-port device after a suitable calibration procedure [4] (match/gain error correction, absolute power accuracy correction, and relative phase calibration correction at different frequencies), mainly when the four scattering parameters must be measured.

In this paper we propose a technique based on the architecture of a feed-forward loop to measure the reflection coefficient of very reflective devices under a fixed-frequency high-power interfering (HPI) signal. Feed-forward techniques have shown to be very useful to measure passive intermodulation (PIM) without using narrowband filters [5], [6]. In those articles, these techniques are applied to cancel the high-power fundamental tones before reaching the spectrum analyzer (SA), which allows increasing the dynamic-range of the PIM measurement system. Since no filters are required, there are no restrictions on the frequency spacing between the driving fundamental tones. Note as well that the measurement system is not specific to a given narrow bandwidth.

To our knowledge, feed-forward techniques have not been previously applied for measuring small-signal scattering parameters under a high-power interfering signal as done on a Hot S-Parameter characterization.

The proposed application for the architecture uses a basic signal cancellation block [7] that requires neither costly external components nor narrowband filters to measure the reflection coefficient of a device under a HPI signal with a traditional VNA, an ENA E5071C in our implementation. The procedure and measurement system described in the paper can be easily extended to measure other scattering parameters of a multiport device.

Section II presents the proposed architecture including the description of the general concept and details on the implementation aspects, such as the cancellation algorithm, which is based on a gradient descent algorithm, and the calibration of the VNA.

Section III applies the implemented measurement system for the characterization of two microwave components, namely a SMA-Female short circuit and a reflective limiter. 


\section{Proposed ARchitecture}

\section{A. General concept}

Fig. 1 outlines the proposed measurement system, where the feed-forward approach can be easily identified by the two existing branches. One branch is used to feed the device under test (DUT) with a single-tone HPI signal, whereas the other branch is used as a reference branch that cancels out the high power reflected signal before reaching the VNA. Both branches are referred, respectively, as DUT-branch and cancellation-branch.

The HPI signal is generated at single frequency $f_{1}$, with a signal generator, which is then followed by an attenuator to adjust the signal power level to the following stages of the system without damaging any of the following components, and by an isolator to guarantee isolation from reflected signals towards the signal generator. The HPI signal goes then through a power splitter to drive the DUT and cancellation branches.

The DUT-branch includes a high-power amplifier (HPA) and an isolator to protect it from reflected signals. The isolator also prevents power-dependent changes of the HPA output impedance that could affect the error terms of the VNA calibration, which is performed at low power levels.

The cancellation-branch is mainly composed of a vector modulator (VM) [7] and a HPA. The attenuation and phase of the VM output signal are controlled by an in-phase voltage $\left(V_{i}\right)$ and a quadrature-voltage $\left(V_{q}\right)$ through a digital to analog converter (DAC). The cancellation signal, with the required phase and magnitude, is then amplified by the HPA followed by an isolator. A variable attenuator is also placed before the $\mathrm{VM}$, which guarantees that the VM works close to the optimum gain state for highest phase resolution.

The amplified signal of the DUT-branch is then connected to a high-power (HP) broadband $90^{\circ}$ hybrid coupler to feed the DUT. The reflected signal from the DUT is directed by the hybrid coupler towards a HP Wilkinson power combiner (labeled A in Fig.1), where it is combined with the signal coming from the cancellation-branch. The magnitude and phase of the cancellation signal is adjusted to be equal in magnitude and opposite in phase than the reflected signal to achieve the desired cancellation level. Note that the combiner must be able to dissipate the power of both signals when they are cancelled.

Then the combiner is connected to the VNA through a HP broadband directional coupler and a power limiter. The HP directional coupler couples the signal to a SA, which is used to measure the power of the cancelled HPI signal. The cancellation level is the input of the algorithm in charge of adjusting the in-phase and quadrature voltages, $V_{i}$ and $V_{q}$, of the VM that modifies the phase and amplitude of the cancellation signal. The required $V_{i}$ and $V_{q}$ values, which are generated with the DAC, are obtained in real time with an iterative algorithm. The only mission of the limiter, whose limiting threshold is $25 \mathrm{dBm}$, is to protect the VNA from a HP signal when cancellation is not achieved. In case of cancellation, the VNA will measure the reflection coefficient of the DUT without the eventual distortion caused by the VNA receivers due to the HPI signal.
All the components and cables dealing with high-power levels (high-power stage in Fig.1) are HP components with $7 / 16$ connectors. Using this configuration, the available input power to the device is $39 \mathrm{dBm}$ (43 dBm HPA are used) and the variable attenuator of the cancellation-branch allows measuring devices with reflection coefficients ranging from 0 to $-50 \mathrm{~dB}$ while keeping both paths properly balanced to allow cancellation. The frequency bandwidth of the VNA measurement path is strictly limited by the bandwidth of the hybrid, combiner, and coupler. In this arrangement the most restrictive is the power combiner with a practical bandwidth from $700 \mathrm{MHz}$ to $2.7 \mathrm{GHz}$.

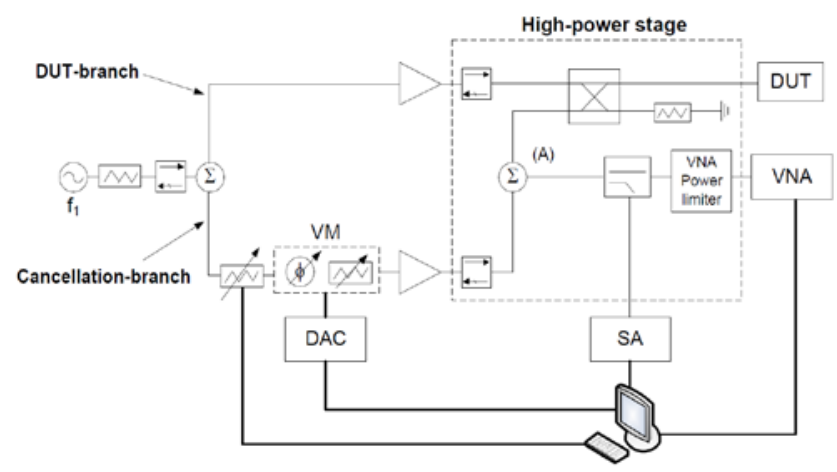

Fig. 1. Feed-forward cancellation set-up.

\section{B. Cancellation algorithm}

As explained in the previous section, the VM is controlled by two DC voltages, $V_{i}$ and $V_{q}$, which set the amplitude and phase of the cancellation signal. The cancellation level $C$ is defined as the ratio of the power of the HPI signal reflected from the DUT without cancellation, to the power of the cancelled signal, both measured at the output of the combiner A (Fig. 1). The un-cancelled power level of reference is measured when the VM is set to its maximum attenuation state.

The power of the cancelled signal depends on the unbalanced phase and magnitude between the signals added in the combiner, so the cancellation level achieves a maximum when both signals are added having equal magnitude and opposite phase.

The values of the required $V_{i}$ and $V_{q}$ are obtained through a cancellation algorithm whose input is the power level measured at the SA. Our cancellation algorithm, which is based on the method of steepest descent in a bi-dimensional domain, is iterative and, therefore, it is well suited for experiments where the reflection coefficient of the device could slightly change with power or time.

If the cancellation level has only a single maximum in the dynamic range of $V_{i}$ and $V_{q}$, the algorithm is able to achieve a desired cancellation level $C$ below this maximum, using a standard iterative gradient search method [8] with line search optimization.

A flow chart of the algorithm is shown in Fig. 2. 


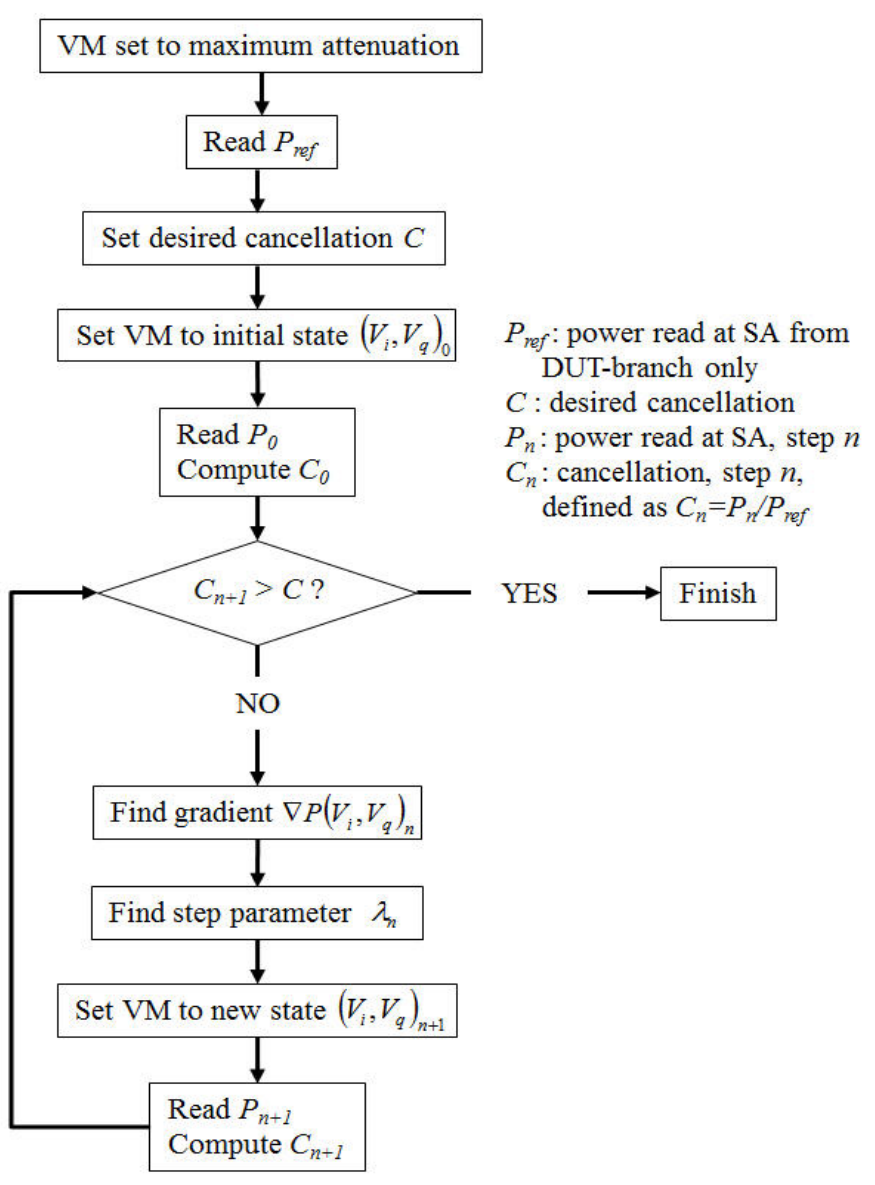

Fig. 2. Gradient search with line optimization algorithm.

The inputs of the algorithm are the power $P$ measured by the SA and the desired cancellation level $C$. The search domain is defined by the voltages, $V_{i}$ and $V_{q}$, that control the vector modulator. Both values are ranged from $0 \mathrm{~V}$ to $1 \mathrm{~V}$ for the $\mathrm{VM}$ used in this setup (Analog Devices AD8341).

The algorithm starts at an initial VM state, $\left(V_{i}, V_{q}\right)_{0}$. A new VM state (given by the pair $V_{i}$ and $V_{q}$ at iteration $n+1$ ) is calculated by computing the gradient of the power $\nabla P$ in the previous modulator state (given by the pair $V_{i}$ and $V_{q}$ at iteration $n$ ) and a scalar step parameter $\lambda_{n}$ as indicated by (1):

$$
\left(V_{i}, V_{q}\right)_{n+1}=\left(V_{i}, V_{q}\right)_{n}-\lambda_{n} \cdot \nabla P\left(V_{i}, V_{q}\right)_{n}, n \geq
$$

The gradient direction is calculated using numerical derivatives. Four power samples are taken in the vicinity of the previous VM state $\left(V_{i}, V_{q}\right)_{n}$ considering a small voltage increment $\Delta$ along the in-phase and quadrature axis:

$$
\begin{aligned}
& P_{-i, n}=P\left(\left(V_{i}, V_{q}\right)_{n}-(\Delta, 0)\right) \\
& P_{+i, n}=P\left(\left(V_{i}, V_{q}\right)_{n}+(\Delta, 0)\right) \\
& P_{-q, n}=P\left(\left(V_{i}, V_{q}\right)_{n}-(0, \Delta)\right) \\
& P_{+q, n}=P\left(\left(V_{i}, V_{q}\right)_{n}+(0, \Delta)\right)
\end{aligned}
$$

The gradient is found by combining these four power samples:

$$
\nabla P\left(V_{i}, V_{q}\right)_{n}=\left(\frac{2 \Delta}{P_{+i, n}-P_{-i, n}}, \frac{2 \Delta}{P_{+q, n}-P_{-q, n}}\right)
$$

In (1) the step parameter $\lambda_{n}$ is obtained to reach the minimum value of the measured power along the gradient direction. This value considers the distance between the power at the current VM state and the power level to achieve the desired cancellation level. The algorithm is stopped during the cancellation process in case of achieving the desired cancellation level. Then the reflection coefficient of the DUT is measured with the VNA.

As an example, Fig. 3 outlines the cancellation transition of a $70 \mathrm{~dB}$ cancellation procedure when measuring a short circuit. It illustrates how the cancellation level increases with the number of measured samples. The starting VM state was $(0 \mathrm{~V}$, $0.5 \mathrm{~V}$ ), near a contour of maximum $\mathrm{VM}$ gain. In the experiment the number of power samples required to achieve a cancellation level of $70 \mathrm{~dB}$ was 127 . This number includes the power samples needed to compute the gradient. The steep valleys in the plot correspond to the samples acquired while computing the gradient and finding a convenient value for the step parameter. The figure in the inset shows the preceding pairs $\left(V_{i}, V_{q}\right)$ commanded to the $\mathrm{VM}$ before reaching the optimum. It reveals that when the cancellation algorithm is close to the desired solution (high cancellation levels) the path followed by the VM state curls towards the cancellation level goal.

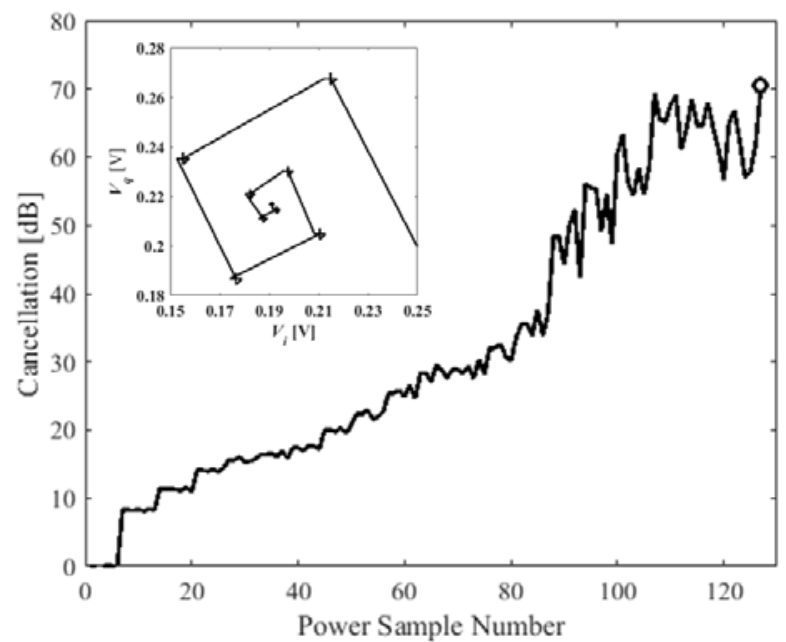

Fig. 3. Example of a $70 \mathrm{~dB}$ cancellation using the gradient search algorithm. It shows the evolution of the cancellation level versus the power sample (including the samples required to compute the gradient). The inset shows the last steps on the evolution of the VM state.

To further illustrate this process, Fig. 4(a) details the cancellation level (left y-axis) and the number of samples required in the cancellation procedure (right $y$-axis) when the cancelling level was set to $70 \mathrm{~dB}$, per one hundred different trials. Those trials were performed when using a short circuit as a DUT.

Fig. 4 (b) is a histogram showing how many assays (vertical axis) achieve the desired cancellation level in each range of 
power samples (horizontal axis). According to this plot, 78 out of 100 assays take less than 200 power samples to get at least $70 \mathrm{~dB}$ of cancellation.

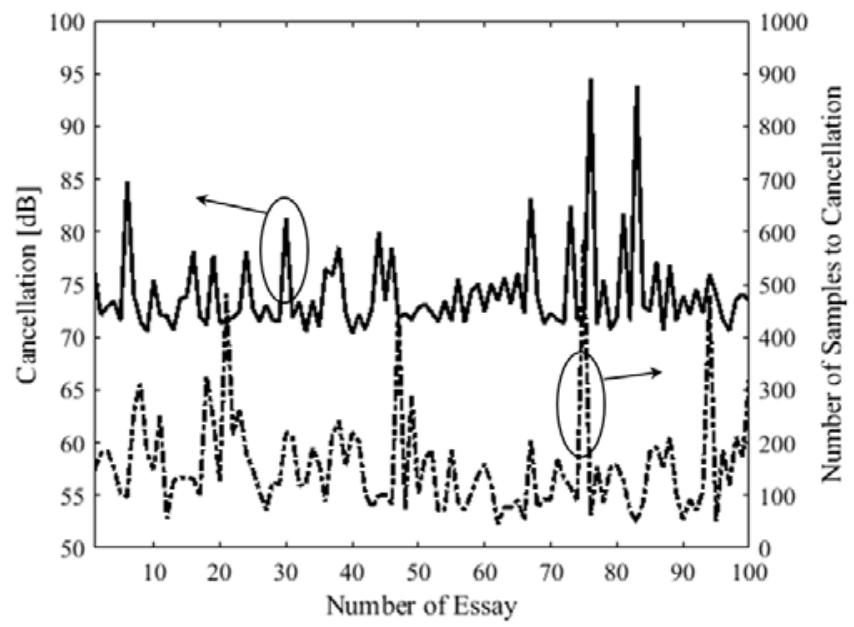

(a)

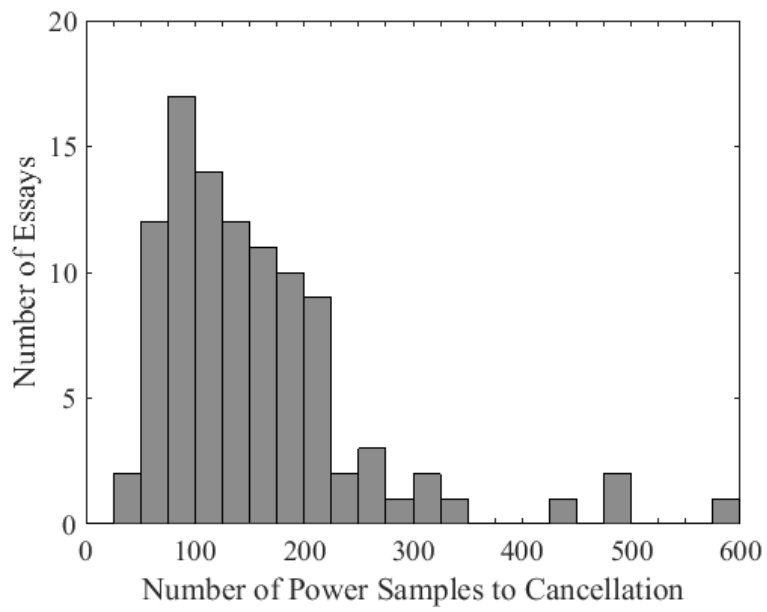

(b)

Fig. 4. One hundred assays of the cancellation algorithm with a $70 \mathrm{~dB}-$ cancellation goal. (a) Left axis shows the cancellation level achieved. The number of samples required to get that level is in the right axis. (b) Histogram showing the number of assays reaching cancellation in a given range of power samples (i.e. convergence speed).

\section{Phase imbalance control in the cancellation-branch}

As outlined in Section II-A, a DAC is used in the cancellation-branch to control the VM through the $V_{i}$ and $V_{q}$ voltages. The quantization error of the DAC is the ultimate limit on the achievable cancellation level [7]. When the signals to be cancelled are balanced in magnitude, the DAC resolution is critical to achieve the phase that cancels out the single tone HP signal.

The vector modulator used in this work is an Analog Devices AD8341 that allows a continuous forward gain magnitude control of $30 \mathrm{~dB}$ starting from a (maximum) $-4.5 \mathrm{~dB}$ and a continuous phase control of $360^{\circ}$. This amplitude and phase control requires single-ended in-phase and quadrature voltages in the range from $0 \mathrm{~V}$ to $1 \mathrm{~V}$. The performance of the $\mathrm{VM}$ is commanded by a National Instruments NI-9264 DAC having 16 bits in a $\pm 10 \mathrm{~V}$ range, that is almost equivalent to 12 bits in a scale of $0-1 \mathrm{~V}(0.3 \mathrm{mV})$. Fig. 5 shows, as an example, the measured magnitude and phase variation of the VM forward gain versus the in-phase and quadrature voltages at a frequency of $2.30 \mathrm{GHz}$. Contours of $-10 \mathrm{~dB}$ and $-15 \mathrm{~dB}$ are indicated in the figure.
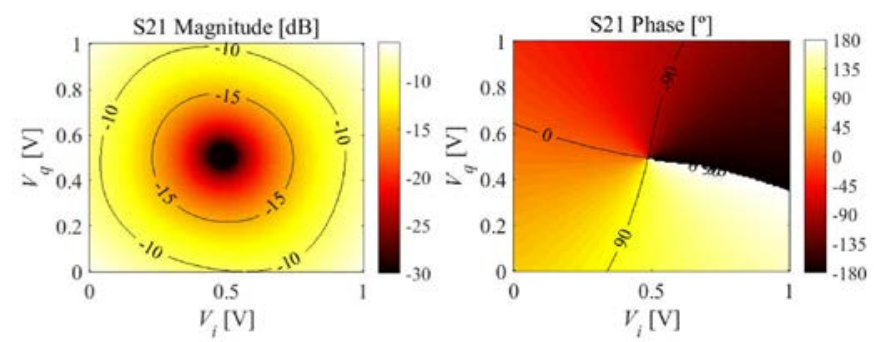

Fig. 5. Measured forward gain $\left(S_{21}\right)$ of the vector modulator at $2.30 \mathrm{GHz}$ with respect to in-phase and quadrature voltages. The $-10 \mathrm{~dB}$ contour allows a full $360^{\circ}$ control.

The VM should provide the required cancelling signal with high accuracy (i. e. close in magnitude and phase of the desired signal). Since the number of voltages $\left(V_{i}, V_{q}\right)$ that can be supplied by the DAC are discrete it is desirable that the VM operates at the highest forward gain, while keeping a full $360^{\circ}$ control of its phase. In the example of Fig. 5, a convenient level for the $S_{21}$ of the vector modulator is $-10 \mathrm{~dB}$ at $2.3 \mathrm{GHz}$. The variable attenuator before the VM helps to set the modulator in this operating condition. Note that setting the value of this attenuator is commonly required only once for a device characterization process.

\section{VNA calibration}

The VNA (Agilent ENA-5071) calibration needs to include all the additional components of the measurement setup up to the reference plane of the DUT. During the calibration process, the active components, VM and HP amplifiers, are turned on to evaluate the noise that they generate. The signal source is switched off to avoid running the cancellation process during the calibration procedure.

With the previous considerations, one-port OSL calibration (standard Agilent 85033D) has been used from $1.8 \mathrm{GHz}$ up to $2.3 \mathrm{GHz}$. The power of the VNA is set to $10 \mathrm{dBm}$ and the IF bandwidth to $1 \mathrm{kHz}$. The swept mode is activated, and four averages are taken at each measurement point. The number of points is set to 1601 points.

Fig. 6 shows the measured $S_{11}$ of the female short circuit of the calibration kit at several power levels of the interfering signal, whose frequency is $1.9 \mathrm{GHz}$, after running the cancellation process. A measurement of the same DUT applying a raw calibration that does not include the measurement system (“CalRef" trace) has been also outlined as a reference. As it can be seen, the reflection coefficient of the DUT does not change significantly at those power levels and the calibration performed at low power levels is still valid. This confirms that the HP components, connectors and cables in the HP stage of the system do not change significantly with the power, and the isolators located at the output of the HPAs offer enough isolation to provide calibration error terms independent of the high-power interfering signal.

The price paid in terms of calibration performance is an 
error around $\pm 0.01 \mathrm{~dB}$ due to the complexity of the system included in the calibration (slow variations with a periodicity of around $0.05 \mathrm{GHz}$ already present at the lowest power) and the noise introduced by the active components: the HPA and VM.
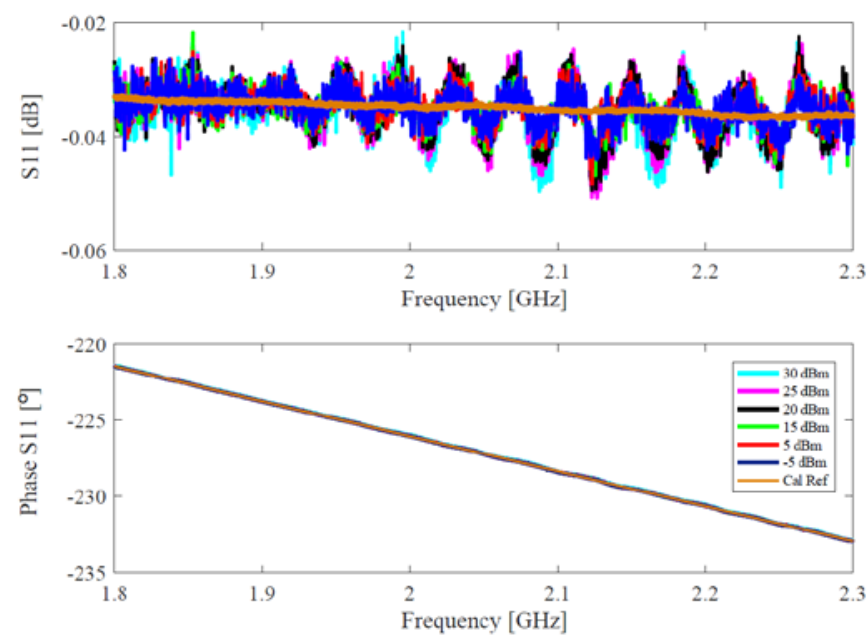

Fig. 6. $S_{11}$ of a SMA-female short measured for a high-power interfering signal from $-5 \mathrm{dBm}$ up to $30 \mathrm{dBm}$. The cancellation algorithm is active.
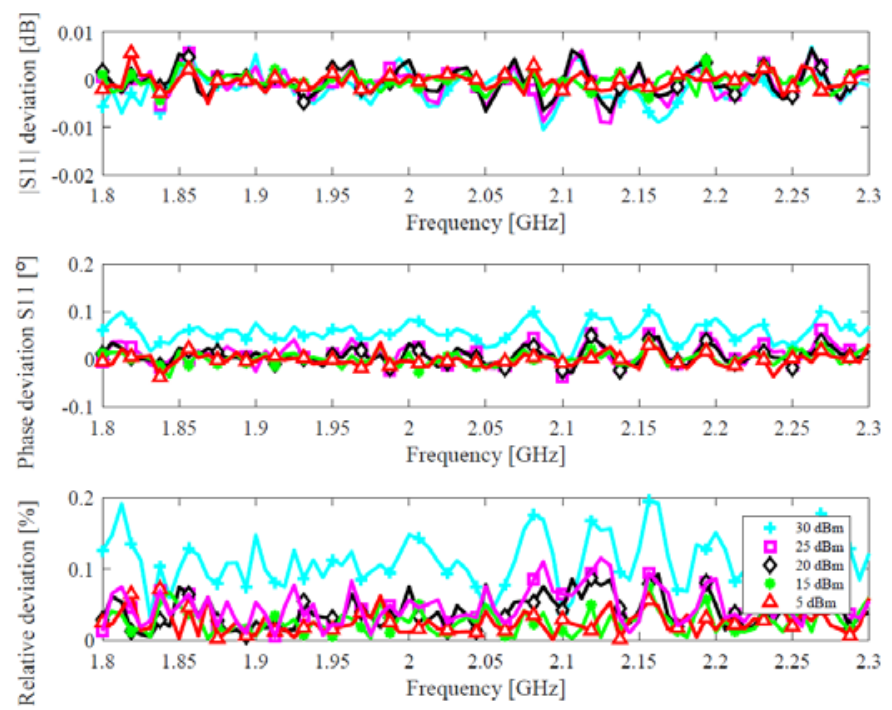

Fig. 7. $S_{11}$ deviation from the $S_{11}$ reference $(-5 \mathrm{dBm})$ of a SMA-female short measured magnitude (top) and phase (middle) for a HPI signal from $5 \mathrm{dBm}$ up to $30 \mathrm{dBm}$. Bottom figure represents the absolute value of the relative deviation.

Fig. 7 represents the deviation of the measured $S_{11}$ from the $S_{11}$ that is measured at the lowest power level of the HPI signal. The deviation of $S_{11}$ magnitude (Fig. 7(a)) increases a little with the power level but always into the range of $\pm 0.01 \mathrm{~dB}$. The phase deviation (Fig. 7(b)) is less than $0.05^{\circ}$ at moderate power levels and $0.1^{\circ}$ at $30 \mathrm{dBm}$. In any case, the relative percent deviation is less than $0.2 \%$ (Fig. $7(\mathrm{c})$ ). Those figures set the limit of the calibration error.

\section{DEVICE MEASUREMENTS}

This section reports on the frequency response of two reflective devices when those are subject to a single-tone highpower interfering signal. Section A describes measurements of the same female short circuit of the previous section, but in this case without applying the cancellation algorithm to illustrate the efficiency of the proposed scheme. Additional measurements performed in a narrower bandwidth are also shown, with and without cancellation. The second device is a reflective limiter and again we will illustrate the effectiveness of applying the cancellation technique and the proposed architecture.

\section{A. Female short circuit}

The first experiment extends the measurements performed in previous section using the same calibration and power levels without applying the cancellation algorithm. Fig. 8 shows that, for even moderate power levels (20 dBm black-diamond trace), the interfering HP signal significantly affects the measurement.

At high-power levels, the interfering signal at $1.9 \mathrm{GHz}$ is clearly displayed at the VNA, but a more important effect is the clear reduction of the reflection coefficient at high-power levels all over the frequency range. One could think that the female short changes drastically due to thermal effects, or that the performance of the limiter that protects the VNA is affected at those power levels, or even that this effect is caused inside the VNA when it is reached by the high-power signal. With regards to the effects of the limiter, a previous set-up of the system confirms that the power reaching the VNA is around $17 \mathrm{dBm}$ when the input power to the DUT is $20 \mathrm{dBm}$ and the cancellation algorithm is not active. At DUT powers below $20 \mathrm{dBm}$ we can discard the limiter as the origin of $S_{11}$ degradation because its threshold limiting power is $25 \mathrm{dBm}$, and degradation of the $S_{11}$ is very significant even at DUT powers much lower than $20 \mathrm{dBm}$. At power levels higher than $20 \mathrm{dBm}$, the protecting limiter may play a role, but it is not the main source of the measurement degradation in the whole range of powers.

By comparing the measurements with cancellation (Fig. 6) and without cancellation (Fig. 8), it indicates that no thermal effects are significant at those power levels and therefore, the $S_{11}$ degradation is originated in the set VNA-limiter due to the incoming high-power level of the interfering signal reaching the VNA. Therefore, one needs to cancel this out to make possible the measurement of the $S_{11}$.

We have also performed a narrower calibration with a bandwidth of $100 \mathrm{MHz}$ around the HP interfering signal. Fig. 9 shows the measurements without cancellation and Fig. 10 shows the measurements when the cancellation algorithm is activated. This exhibits a significant variation, and the device can be properly measured when the cancellation is applied. The conclusions are the same as those discussed previously and note that an additional peak at $6 \mathrm{MHz}$ below $1.9 \mathrm{GHz}$ in unveiled. By measuring the spectrum of the input signal at the VNA port plane, we confirmed that this signal is not directly 
coming from any interference, intermodulation or other nonlinear effect of the measurement system. Therefore, it must correspond to an intermodulation or harmonic signal generated by the VNA appearing when there is no cancellation of the high-power signal.
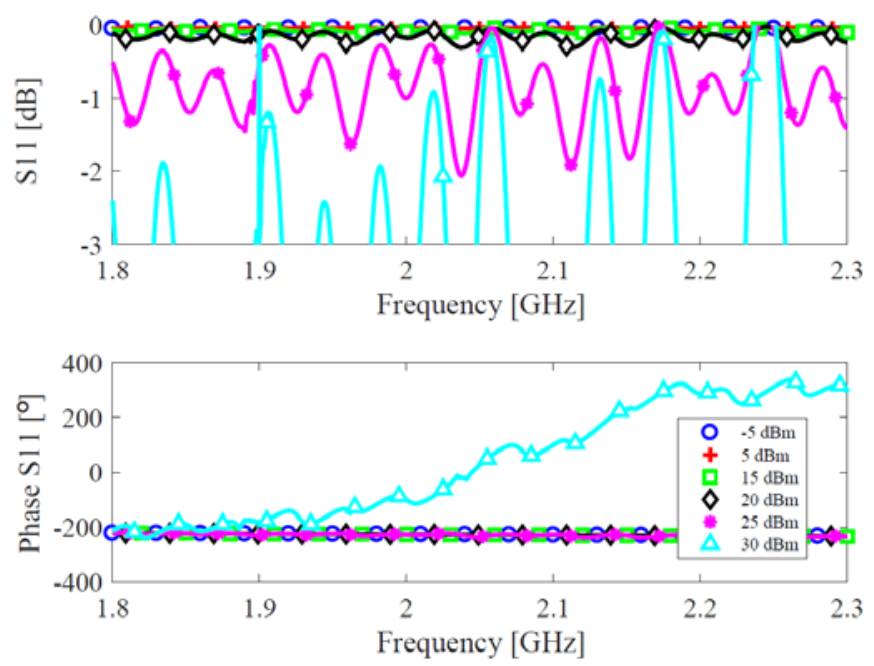

Fig. 8. $S_{11}$ of a SMA-female short measured for a high-power interfering signal from $-5 \mathrm{dBm}$ up to $30 \mathrm{dBm}$. The cancellation algorithm is not active.
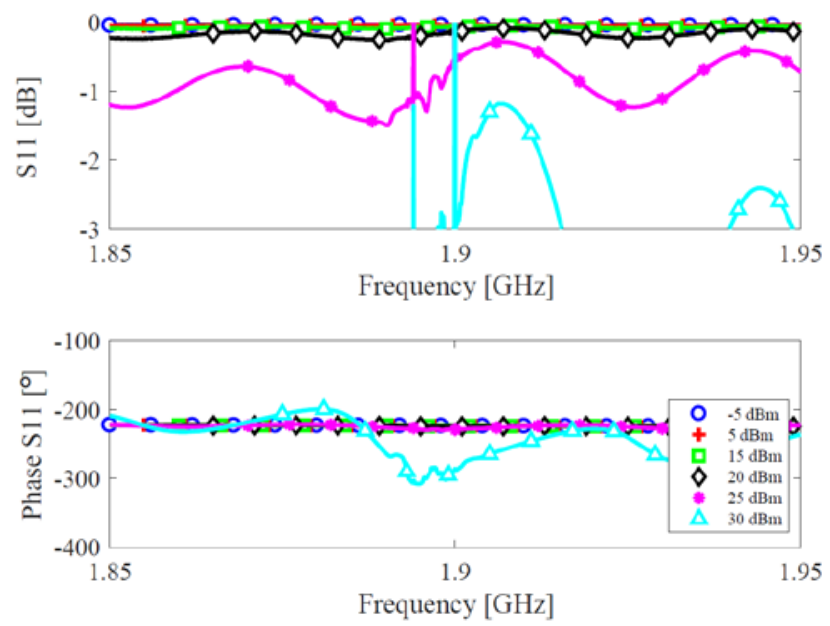

Fig. 9. $S_{11}$ of a SMA-female short measured for a high-power interfering signal from $-5 \mathrm{dBm}$ up to $30 \mathrm{dBm}$. The cancellation algorithm is not active.

Note that in Fig. 10 there is no sign of the HP signal tone in the measurements as it was expected.

We can confirm therefore that the large reduction of $S_{11}$, observed in previous experiments without cancellation algorithm (Fig. 8 and Fig. 9), was due to saturation effects of the circuitry of the VNA-limiter and the performance of the DUT is not affected by the high-power interfering signal. That means that below $30 \mathrm{dBm}$, heating effects in this device are negligible.

\section{B. Reflective limiter}

A high-power limiter (LM200802-M-A-300) has been measured. The output port of the device was terminated with a high-power $50 \Omega$ load. The input power level of the $1.9 \mathrm{GHz}$ high-power interfering signal was increased from $-5 \mathrm{dBm}$ up to $30 \mathrm{dBm}$, reaching $35 \mathrm{dBm}$ when the cancellation is active.
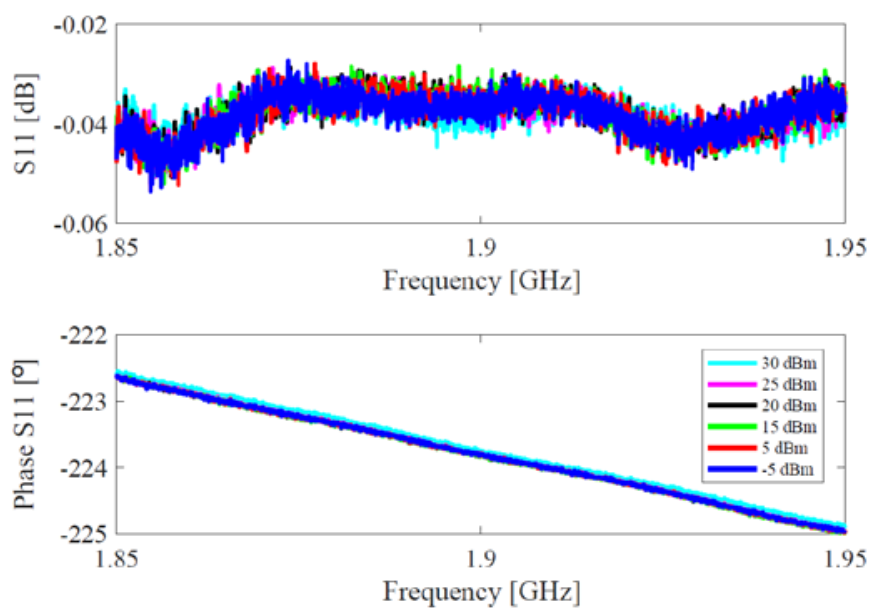

Fig. 10. $S_{11}$ of a SMA-female short measured for a high-power interfering signal from $-5 \mathrm{dBm}$ up to $30 \mathrm{dBm}$. The cancellation algorithm is active.
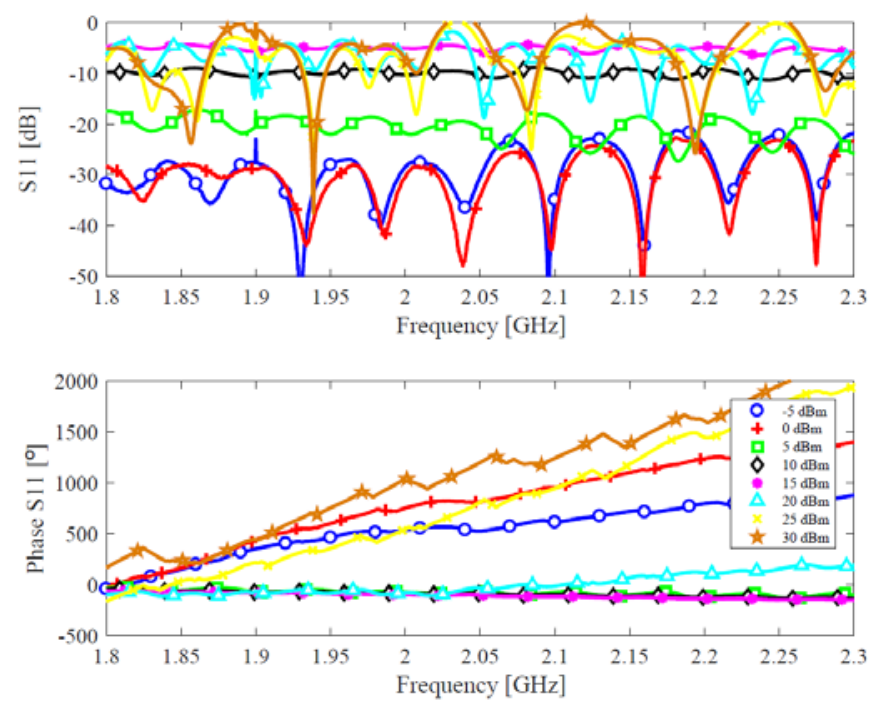

Fig. 11. Measured $S_{11}$ of the limiter without cancellation for several interfering power levels.

Results for the non-cancellation and cancellation cases are reported in Fig. 11 and Fig. 12, respectively. The reflection coefficient goes from the all-pass state to the limiting state losing the good return losses as corresponds to a reflective limiter. Note that for the cancellation case, the cancellation algorithm must be applied even when the return losses are still high, and the reflected power is low.

Differences between results in Fig. 11 and Fig. 12 reveal that cancellation of the single-tone high-power signal before reaching the VNA is necessary to obtain the reflection coefficient of the DUT.

This statement is further validated with the results in Fig. 13. Fig. 13 uses the reflection coefficients of Fig. 11 and Fig. 12 , to outline the reflection coefficients at two frequency points, $1.9 \mathrm{GHz}$ and $2 \mathrm{GHz}$, as a function of the input power. 
Note that the first frequency point is the frequency where the single-tone high-power signal is set. Results at $1.9 \mathrm{GHz}$ are indicated in blue circles and results at $2 \mathrm{GHz}$ in black triangles. Results corresponding to the non-cancellation case are indicated in dashed-dot traces, whereas the cancellation cases are indicated with solid traces.
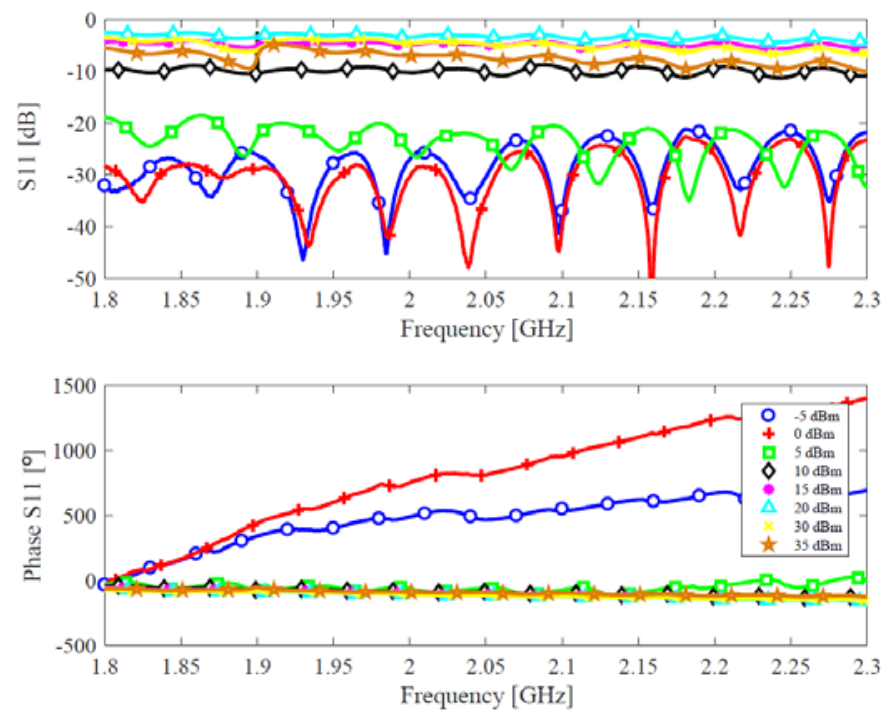

Fig. 12. Measured $S_{11}$ of the limiter with cancellation for several interfering power levels.

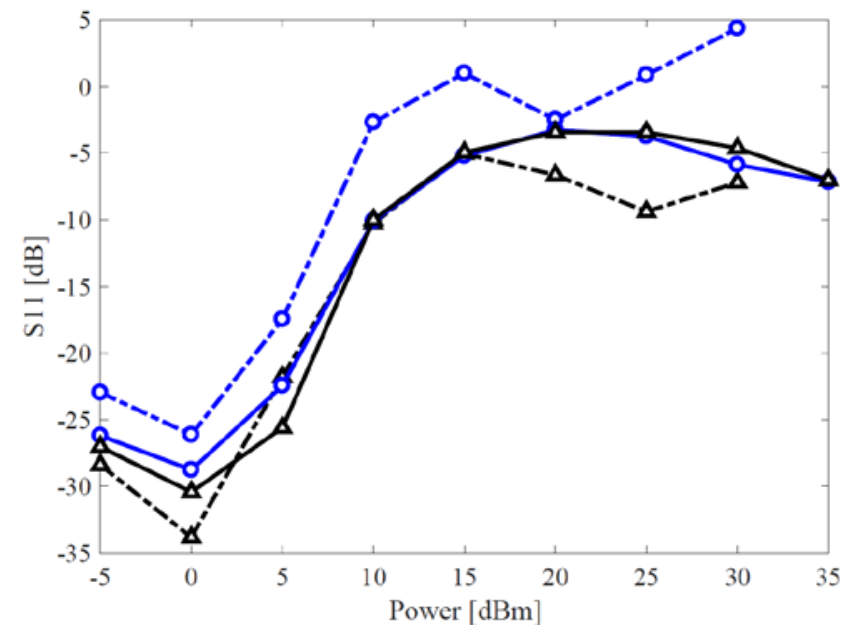

Fig. 13. Return losses of the limiter at $1.9 \mathrm{GHz}$ (blue circles) and $2 \mathrm{GHz}$ (black triangles) with cancellation (solid traces) and without cancellation (dashed-dot traces).

\section{CONCLUSION}

This paper demonstrates that when a device is driven by a high-power interfering signal the proposed feed-forward architecture to measure small signal scattering parameters, in this case the $S_{11}$, is very useful without requiring a HP standard to calibrate the VNA.

To our knowledge, this is the first time that the feed-forward techniques are used to measure small-signal parameters of devices, which are subject to high-power levels of signals. For example, this technique could be used to measure, with a simple VNA, Hot-S22 parameters using neither expensive components, nor complex calibration procedures.

The proposed architecture is especially suited for measuring the reflection coefficient of reflective devices at frequencies close to the frequency of the HPI signal. Once proved the viability of the concept, the system could be easily modified to measure transmission parameters of a two-port device, just adding a new cancellation branch.

\section{REFERENCES}

[1] C. Collado et al., "Absorptive Limiter for Frequency-Selective Circuits", IEEE Microw. Wireless Compon. Lett., vol. 24, no. 6, pp. 415-417, Jun. 2014.

[2] Agilent Technologies (2007, April). “Advanced Measurement Techniques for RF Amplifiers Using Unique Functions of the Agilent E5071C ENA" (Application Note). Agilent Technologies. USA. http://www.testunlimited.com/pdf/an/5989-6522EN.pdf

[3] D. E. Root, J. Verspecht, J. Horn and M. Marcu, X-Parameters. Characterization, modeling, and design of nonlinear $R F$ and microwave components, Cambridge, UK, Cambridge University Press, 2013.

[4] K. F. Anderson, "A Survey of Techniques for Improving the Calibration of High-Power Network Analyzers", 78th ARFTG Microwave Measurement Conference, Tempe, AZ, 2011, pp. 1-5.

[5] J. Mateu, J. C. Booth and S. A. Schima, "Frequency Tuning and Spurious Signal Generation at Microwave Frequencies in Ferroelectric $\mathrm{SrTiO}_{3}$ Thin-Film Transmission Lines", IEEE Trans. Microw. Theory Techn. vol. 55, no. 2, pp. 391-396, Feb. 2007

[6] J. R. Wilkerson, K. G. Gard and M. B. Steer, "Automated Broadband High-Dynamic-Range Nonlinear Distortion Measurement System", IEEE Trans. Microw. Theory Techn., vol. 58, no. 5, pp. 1273-1282, May 2010.

[7] J. M. Wetherington and M. B. Steer, "Robust Analog Canceller for High-Dynamic-Range Radio Frequency Measurement," IEEE Trans. Microw. Theory Techn., vol. 60, no. 6, pp. 1709-1719, Jun. 2012.

[8] G. Lasser, R. Langwieser and C.F. Mecklenbräuker, "Automatic Leaking Carrier Canceller Adjustment Techniques” J. Embedded Systems, no. 8, 2013, https://doi.org/10.1186/1687-3963-2013-8. 